\title{
Regulation of flowering time in chrysanthemum by the R2R3 MYB transcription factor CmMYB2 is associated with changes in gibberellin metabolism
}

\author{
Lu Zhu', Yunxiao Guan', Yanan Liu', Zhaohe Zhang', Muhammad Abuzar Jaffar', Aiping Song', Sumei Chen', \\ Jiafu Jiang ${ }^{1}$ and Fadi Chen ${ }^{1}$
}

\begin{abstract}
The switch from vegetative growth to reproductive growth is a key event in the development of a plant. Here, the product of the chrysanthemum gene CmMYB2, an R2R3 MYB transcription factor that is localized in the nucleus, was shown to be a component of the switching mechanism. Plants engineered to overexpress CMMYB2 flowered earlier than did wild-type plants, while those in which CMMYB2 was suppressed flowered later. In both the overexpression and RNAi knockdown plants, a number of genes encoding proteins involved in gibberellin synthesis or signaling, as well as in the response to photoperiod, were transcribed at a level that differed from that in the wild type. Both yeast two-hybrid and bimolecular fluorescence complementation assays revealed that CmMYB2 interacts with CmBBX24, a zinc-finger transcription factor known to regulate flowering by its influence on gibberellin synthesis.
\end{abstract}

\section{Introduction}

The switch from vegetative growth to reproductive growth is a major developmental event in the life cycle of a flowering plant. This switch is coordinated by the products of a suite of genes that differentially respond to the photoperiod, temperature, and the tissue content of several phytohormones ${ }^{1}$. The successful isolation of a number of such genes has led to the recognition that flowering can be triggered by proteins active in one or more of the photoperiod, gibberellin (GA), vernalization, autonomous, and senescence pathways ${ }^{2-6}$. These pathways come together to form a complex regulatory network that ultimately induces the key floral meristem genes $A P E$ TALA1 (AP1) and LEAFY $(L F Y)^{4-6}$.

A number of MYB transcription factors participate in the regulation of flowering time. The following examples all relate to the model angiosperm species Arabidopsis

\footnotetext{
Correspondence: Jiafu Jiang (jiangjiafu@njau.edu.cn) or

Fadi Chen (chenfd@njauedu.cn)

${ }^{1}$ State Key Laboratory of Crop Genetics and Germplasm Enhancement, Key Laboratory of Landscaping, Ministry of Agriculture, College of Horticulture, Nanjing Agricultural University, Nanjing, China
}

thaliana. Plants engineered to overexpress the $M Y B$ related gene CIRCADIAN CLOCK ASSOCIATED 1 (CCA1) are compromised with respect to their recognition of the circadian clock and consequently suffer from a delay in flowering ${ }^{7}$. Overexpression of the gene EARLYPHYTOCHROME-RESPONSIVE 1 (EPR1) enhances responsiveness to far-red light, which also leads to delayed flowering ${ }^{8}$. Overexpression of CAPRICE 3 (CPL3) downregulates several key flowering time genes, including FLOWERING LOCUS T (FT), SUPPRESSOR OF OVEREXPRESSION OF CO1 (SOC1) and CONSTANS (CO $)^{9}$. The product of WEREWOLF (WER) is known to act in the photoperiod pathway, since flowering is delayed in the wer mutant when exposed to long-day conditions ${ }^{10}$. MYB30 interacts with the $F T$ promoter to promote flowering ${ }^{11}$. EARLY FLOWERING MYB PROTEIN (EFM) is involved in both the photoperiod and the temperature flowering pathways $^{12}$, while PHLOEM DEVELOPMENT (APL) (syn. PE) activates $F T^{13}$. The flowering of plants overexpressing MYB44 is delayed by 6-7 days ${ }^{14}$. MYB transcription factors that affect flowering are also present in other species. For example, the product of the poplar gene

\section{(c) The Author(s) 2020}

(c) Open Access This article is licensed under a Creative Commons Attribution 4.0 International License, which permits use, sharing, adaptation, distribution and reproduction cc) in any medium or format, as long as you give appropriate credit to the original author(s) and the source, provide a link to the Creative Commons license, and indicate if changes were made. The images or other third party material in this article are included in the article's Creative Commons license, unless indicated otherwise in a credit line to the material. If material is not included in the article's Creative Commons license and your intended use is not permitted by statutory regulation or exceeds the permitted use, you will need to obtain permission directly from the copyright holder. To view a copy of this license, visit http://creativecommons.org/licenses/by/4.0/. 

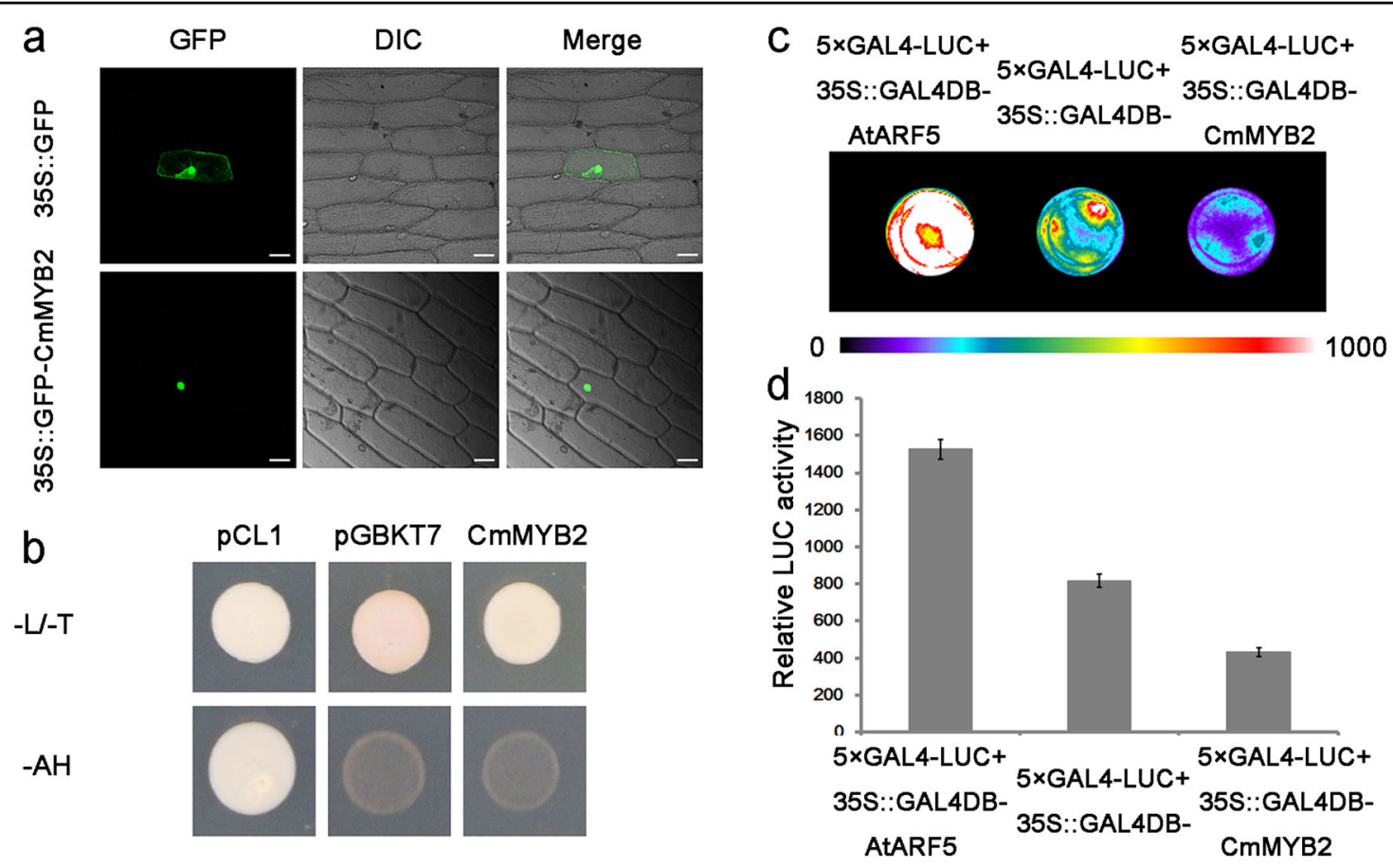

Fig. 1 Subcellular localization and transactivation activity of CmMYB2. a Site of CmMYB2 deposition in transiently transformed onion epidermal cells. Bar: $50 \mu \mathrm{m}$. b Transcriptional activation activity of CMMYB2 in yeast. PCL1 and PGBKT7 plasmids were used as the positive and negative controls, respectively. -L/-T: SD media lacking leucine/SD media lacking tryptophan, -AH: SD media lacking both histidine and adenine. c, $\mathbf{d}$ Luciferase activity in A. thaliana mesophyll protoplasts expressing 5xGAL4-LUC together with either p355::GAL4DB-AtARF5, p355::GAL4DB or p35S::GAL4DB-CmMYB2. c Arabidopsis mesophyll protoplasts imaged after luciferin addition with a charge-coupled device (CCD) camera. $\mathbf{d}$ Luciferase activity measured after the introduction of p35S::GALADB-CMMYB2 into A. thaliana mesophyll protoplasts

PtrMYB192 negatively affects flowering time, and its constitutive expression in A. thaliana also induces a delay in flowering ${ }^{15}$. Constitutive expression of the wheat gene TaMYB72 in rice delays flowering time by 12 days ${ }^{16}$. In alfalfa, MsSPL13 downregulates MsMYB112, thereby controlling both vegetative and reproductive development ${ }^{17}$. In chrysanthemum (Chrysanthemum morifolium), a substantial number of MYB transcription factors have been shown to participate in the determination of flowering time ${ }^{18,19}$, although little is known about their specific functions.

Chrysanthemum occupies a large share of the global market of cut flowers ${ }^{20,21}$. Most chrysanthemum varieties are short-day plants and blossom during a relatively short period. The $C m M Y B 2$ gene encodes an R2R3 MYB transcription factor; when this gene is constitutively expressed in A. thaliana, flowering is delayed, and all $C O, F T, S O C 1, L F Y$, and $A P 1$ genes are downregulated $^{22}$. However, in this study, when $C m M Y B 2$ is overexpressed in chrysanthemum, the flowering time is promoted, while RNAi-enabled knockdown delays flowering. The regulatory mechanism of flowering was further analyzed via transcriptome sequencing. CmMYB2 was found to interact with CmBBX24, which is a zinc-finger transcription factor that has been shown to alter GB synthesis and regulate flowering time ${ }^{23}$. Thus, the aim of the present study was to characterize in detail the molecular effect of manipulating the expression of $C m M Y B 2$. The results showed that $C m M Y B 2$ may regulate flowering by inhibiting the activity of the CmBBX24 protein and modulating the GA pathway in chrysanthemum.

\section{Results}

\section{Subcellular localization and transactivation activity of CmMYB2}

The sequences of the $C m M Y B 2$ open reading frame present in the varieties Jinba and Zhongshanzigui were identical stretches of $948 \mathrm{nt}$, predicted to encode a 315 residue polypeptide. The polypeptide sequence included an R2R3 MYB domain within its $\mathrm{N}$-terminal region and the characteristic GxFMxVVQEMIxxEVRSYM motif within its C-terminal region ${ }^{22}$. The site of expression of $C m M Y B 2$ was deduced by transiently expressing a transgene comprising the CmMYB2 coding sequence fused to GFP and driven by the CaMV $35 \mathrm{~S}$ promoter in onion epidermal cells. GFP activity was concentrated in the nuclei, while in control transgenic plants harboring the p35S::GFP construct, it was dispersed throughout the nucleus and cytoplasm (Fig. 1a). 
To determine whether CmMYB2 can function as a transcription factor, a transactivation assay was performed in yeast. Yeast cells harboring pCL1 were able to grow freely on synthetic dropout (SD) media lacking histidine and adenine, while cells harboring pGBKT7 were able to grow only on SD media lacking tryptophan. Yeast cells harboring $C m M Y B 2$ were unable to activate either the His3 or the Ade2 reporter gene, preventing growth on SD media lacking histidine and adenine (Fig. 1b). The results suggested that CmMYB2 showed no transactivation activity in yeast. A series of equivalent assays was attempted in $A$. thaliana protoplasts, in which the positive control was p35S::GAL4DB-ARF5 and in which the negative control was $\mathrm{p} 355:: G A L 4 D B$. The reduction in LUC activity exhibited by protoplasts harboring C $m M Y B 2$ compared to the activity in protoplasts harboring $\mathrm{p} 35 \mathrm{~S}:$ : GAL4DB suggested that CmMYB2 could act as a transcriptional repressor in $A$. thaliana protoplasts (Fig. 1c).

\section{Effects of manipulating the expression of $C m M Y B 2$ on plant growth and development}

Both CmMYB2 overexpression (OX lines) and $C m M Y B 2$ knockdown (RNAi lines) were generated, and their growth and development were monitored at various time points (Fig. 2a). The flowering time of both the OX and RNAi plants differed significantly from that of wildtype (WT) plants. At 80 days after transplanting, OX plants had formed visible flower buds and had entered the flower bud development stage (FBD); whereas this event occurred at 95 days in WT plants and at 99 days in RNAi plants. The differentiation of the first flower buds and beginning of the petal primordium differentiation stage occurred 15 days sooner in OX plants than in WT plants, while the RNAi plants took an additional four days to reach this stage. By 112 days after transplanting, the flowers of OX plants had entered the early opening stage (EO); however, those formed by WT plants did not reach this stage until 127 days after transplantation, and those formed by RNAi plants did not reach it until 130 days after transplantation (Fig. 2b-d). There were no significant differences among the OX, RNAi and WT plants with respect to their overall height, leaf and flower number or diameter of their flowers.

\section{Transcription profiling of genes acting downstream of CmMYB2 and associated with flowering time}

RNA-seq was implemented to identify differential transcription between WT, OX and RNAi plants (Fig. 3a); Venn diagrams were constructed to show the distribution of these genes visually (Fig. 3b, c). The genes

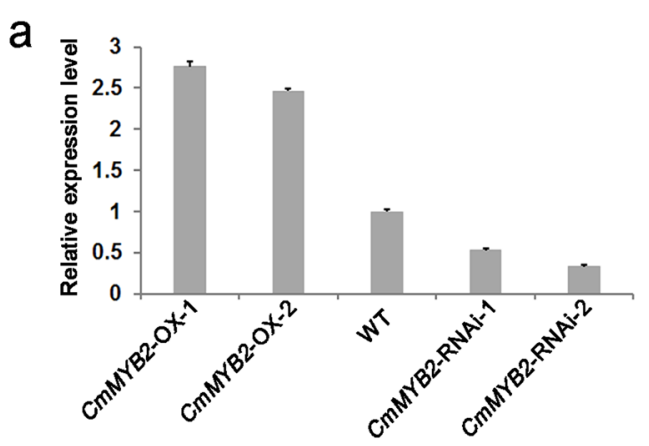

C

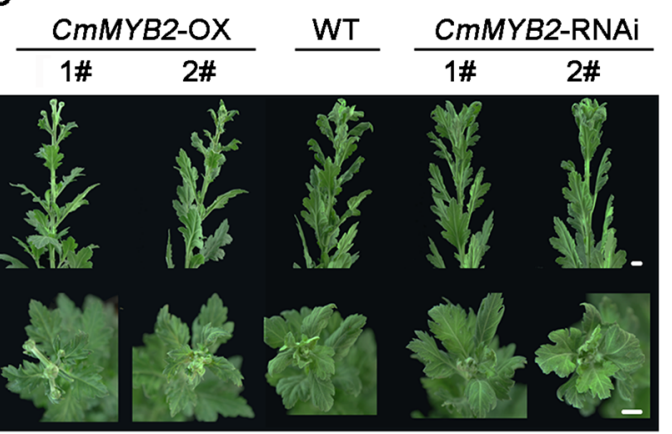

b
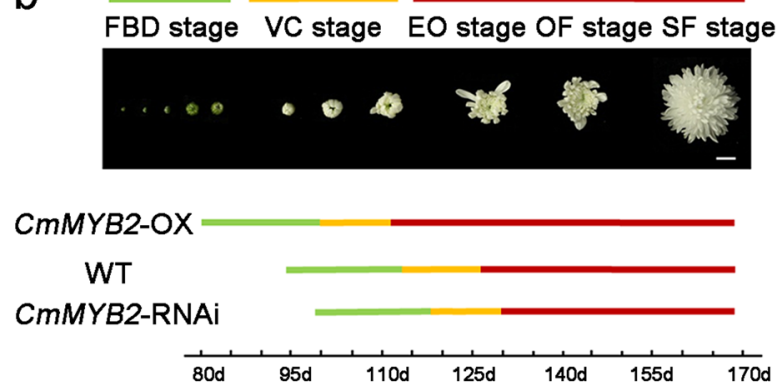

d
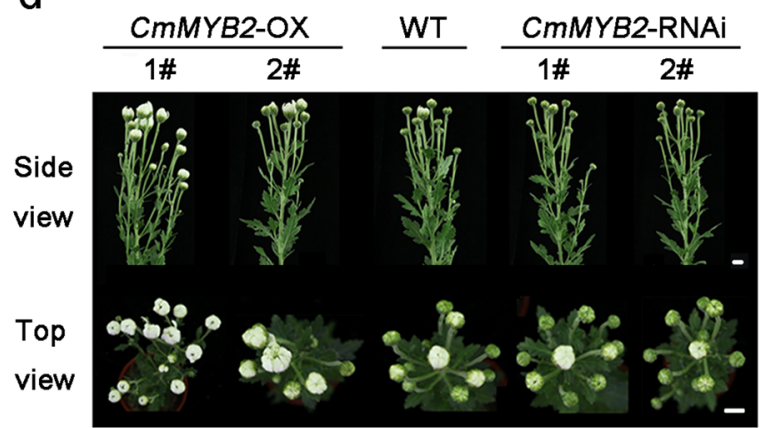

Fig. 2 Phenotypic characterization of CmMYB2-overexpressing (OX) and CmMYB2 knockdown (RNAi) chrysanthemum plants. a $q R T-P C R$ analysis of CMMYB2 transcription in WT, OX and RNAi plants. OX-1, 2: two independent CmMYB2 overexpressors, RNAi-1, -2: two independent CMMYB2 RNAi knockdowns. The values are shown as the mean \pm SEs $(n=3)$. b Development of flower buds in transgenic and WT plants. FBD: flower bud development stage, VC: visible color stage, EO: early opening stage, OF: open-flower stage, SF: senescent-flower stage. Bars: $1 \mathrm{~cm}$. c, d Phenotypes of WT, OX and RNAi plants at the reproductive stage. Plants were imaged at (c) 100 days and (d) 120 days after transplanting 


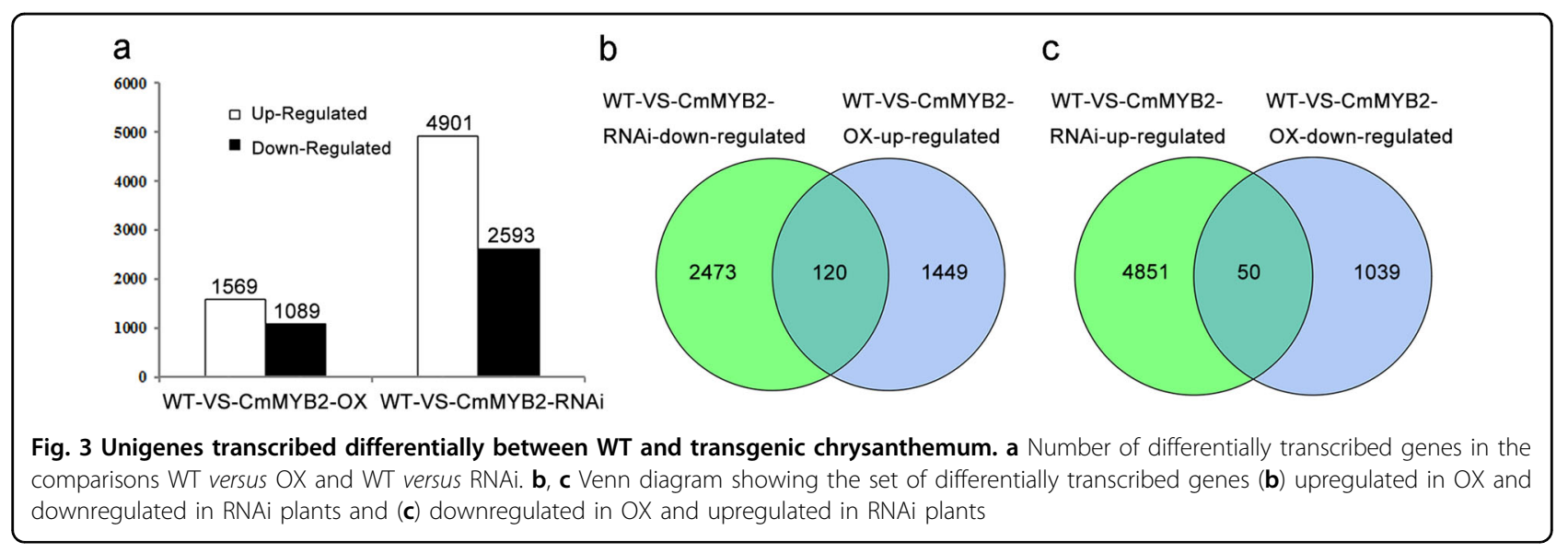

that were either more strongly transcribed in the OX plants than in the WT plants but weakly transcribed in the RNAi plants or vice versa were emphasized. Among the large number of such genes identified, many of their encoded proteins were involved in either the GA synthesis/signaling pathway or the photoperiod pathway; of particular interest were CmGA20ox, CmGA2ox, CmGA3ox, CmGRP, CmGID, and CmDELLA (the GA synthesis and signaling pathway) as well as CmCOL1, CmCOL9, CmPRR7, CmPRR9, and CmCDF1 (the photoperiod pathway) (Table 1). The results of the quantitative real-time PCR (qRT-PCR) assays used to confirm the RNA-seq-based identification are shown in Fig. 4. The analysis confirmed that CmGA20ox (Unigene70017), CmGA2ox (Unigene15204), CmGA3ox (CL3299.Contig1 and Unigene25287), CmGRP (Unigene8840 and CL1665.Contig2), CmGID1 (Unigene22516), CmCOL1 (CL3612.Contig1) and CmPRR7 (CL12170.Contig2) were more strongly transcribed in OX plants than in WT plants but were more weakly transcribed in RNAi plants; moreover, the abundance of CmDELLA (Unigene14903, CL5826.Contig1 and Unigene26087), CmCOL9 (CL11719.Contig1) and CmCDF1 (CL3093.Contig2 and CL3391.Contig1) transcripts was lower in the OX plants than in the WT plants, but it was higher in the RNAi plants.

\section{CmMYB2 alters cellular GA contents through its interaction with $C m B B \times 24$}

Quantification of the $\mathrm{GA}_{1}, \mathrm{GA}_{19}$, and $\mathrm{GA}_{20}$ contents of young leaves of WT, OX and RNAi plants grown under short days showed that these compounds were significantly enhanced in OX plants and significantly reduced in RNAi plants (Table 2). A yeast two-hybrid screen was then performed to identify the proteins able to interact with CmMYB2. CmMYB9A was found to be a potential interacting protein with CmMYB2, and CmBBX24 was also found to interact with CmMYB9A
Table 1 Differentially transcribed genes associated with flowering time

\begin{tabular}{|c|c|c|c|}
\hline \multirow[t]{2}{*}{ Gene } & \multirow[t]{2}{*}{ Annotation } & \multicolumn{2}{|c|}{$\begin{array}{l}\text { Fold Change } \\
\text { (log2 } \\
\text { transformed) }\end{array}$} \\
\hline & & OX/WT & RNAi/WT \\
\hline \multicolumn{4}{|c|}{ GA biosynthesis and signaling } \\
\hline Unigene70017_All & GA 20-oxidase & 1.0 & -5.4 \\
\hline Unigene15204_All & GA 2-oxidase & 0.7 & -1.0 \\
\hline CL3299.Contig1_All & GA 3-oxidase 1-like & 0.3 & -1.5 \\
\hline Unigene25287_All & GA 3-oxidase 4 & 0.3 & -2.3 \\
\hline Unigene8840_All & $\begin{array}{l}\text { Gibberellin-regulated family } \\
\text { protein }\end{array}$ & 2.0 & -0.7 \\
\hline CL1665.Contig2_All & $\begin{array}{l}\text { Gibberellin-responsive } \\
\text { protein }\end{array}$ & 0.2 & -4.6 \\
\hline Unigene22516_All & Gibberellin receptor GID1 & 0.6 & -1.3 \\
\hline Unigene14903_All & DELLA protein & -0.3 & 1.9 \\
\hline CL5826.Contig1_All & DELLA protein & -3.5 & 0.3 \\
\hline Unigene26087_All & DELLA protein & -0.4 & 1.0 \\
\hline \multicolumn{4}{|l|}{ Photoperiod pathway } \\
\hline CL3612.Contig1_All & CONSTANS-LIKE 1 & 1.6 & -1.1 \\
\hline CL11719.Contig1_All & CONSTANS-LIKE 9 & -1.1 & 1.5 \\
\hline CL12170.Contig2_All & $\begin{array}{l}\text { PSEUDO-RESPONSE } \\
\text { REGULATOR } 7\end{array}$ & 0.6 & -1.1 \\
\hline CL3093.Contig2_All & CYCLING DOF FACTOR 1 & -1.2 & 0.6 \\
\hline CL3391.Contig1_All & CYCLING DOF FACTOR 1 & -0.9 & 1.5 \\
\hline
\end{tabular}

The thresholds for significance were $P<0.05$ and FDR $\leq 0.001$

WT wild type, OX CmMYB2 overexpression, RNAi CmMYB2 knockdown

(Table S2). This implied that CmMYB2 may directly interact with CmBBX24. CmBBX24 contained two Bboxes at the N-terminus. $C m B B X 24 b o x s$ was two B-boxes 

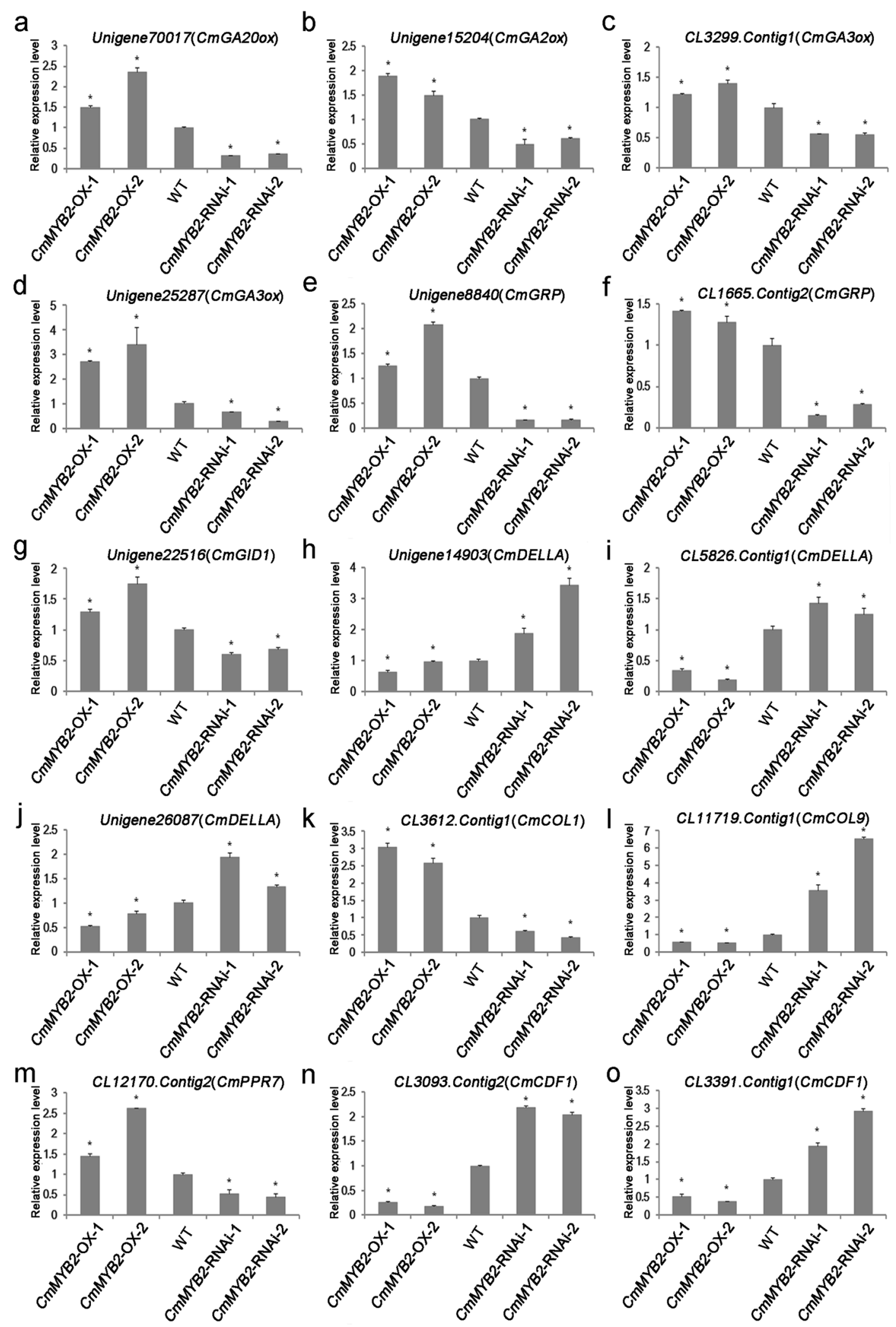

Fig. 4 qRT-PCR validation of differential transcription predicted by RNA-seq. a CmGA200x, b CmGA20x, c CmGA30x, d CmGA30x, e CmGRP, $\mathbf{f} C m G R P, \mathbf{g} C m G I D 1, \mathbf{h} C m D E L L A, \mathbf{i} C m D E L L A, \mathbf{j} C m D E L L A, \mathbf{k} C m C O L 1, \mathbf{I} C m C O L 9, \mathbf{m} C m P R R 7, \mathbf{n} C m C D F 1, \mathbf{o} C m C D F 1$. The values are shown as the mean \pm SEs $(n=3)$. The means for each gene followed by the same letter do not differ significantly from one another $(P<0.05)$ 
at the $\mathrm{N}$-terminus of $C m B B X 24$ and $C m B B X 24 b o x 2$ was the second B-box at the N-terminus of $C m B B X 24$. Yeast cells cotransformed with $C m M Y B 2$ and $C m B B X 24 b o x s$ were able to grow on media lacking tryptophan, leucine, histidine and adenine, regardless of whether X- $\alpha$-gal was supplied (Fig. 5a). Additional experiments showed that CmMYB2 was able to interact with CmBBX24box2 (Fig. 5a). A bimolecular fluorescence complementation (BiFC) assay was used to provide experimental evidence that CmMYB2 could interact with CmBBX24 in vivo. In onion epidermal cells transiently transformed with CmMYB2/ cYFP and $C m B B X 24 / n Y F P$ fusion constructs, YFP fluorescence was detected within the nuclei (Fig. 5b), confirming an in vivo interaction between CmMYB2 and CmBBX24.

Table 2 GA content in the leaves of WT and transgenic (OX and RNAi) chrysanthemum plants grown under shortday conditions

\begin{tabular}{llll}
\hline \multicolumn{5}{c}{$\begin{array}{l}\text { GA content (ng } \\
\left.\mathbf{g}^{-\mathbf{1}} \mathbf{F W}\right)\end{array}$} & & \\
\hline Lines & $\mathrm{GA}_{1}$ & $\mathrm{GA}_{19}$ & $\mathrm{GA}_{20}$ \\
WT & $2.78 \pm 0.33$ & $4.85 \pm 0.74$ & $10.41 \pm 0.33$ \\
CmMYB2-OX-1 & $3.22 \pm 0.41^{*}$ & $5.70 \pm 0.92^{*}$ & $12.39 \pm 0.19^{* *}$ \\
CmMYB2-RNAi-1 & $1.46 \pm 0.16^{* *}$ & $2.34 \pm 0.32^{* *}$ & $6.70 \pm 0.48^{* *}$ \\
\hline
\end{tabular}

SD: $8 \mathrm{~h}$ photoperiod. The values are shown as the mean \pm SEs $(n=3) .{ }^{*}$ and ${ }^{* *}$ represent significant differences $(P<0.05,<0.01) . \mathrm{GA}_{4}, \mathrm{GA}_{9}$ and $\mathrm{GA}_{24}$ were not detected

\section{Discussion}

CmMYB2 is expressed in the nucleus and acts as a transcriptional repressor

A common feature of MYB transcription factors is their nuclear localization signal peptides ${ }^{24-26}$. The rice gene OsMYB511 is expressed specifically in the nucleus ${ }^{24}$, as are the Jatropha curcas gene JcR1MYB1 $1^{25}$ and the Nicotiana benthamiana gene NbPHAN ${ }^{26}$. Experiments have shown that for NbPHAN, the N-terminal region is responsible for its nuclear localization ${ }^{26}$. Here, a transient expression experiment based on onion epidermal cells was able to show that CmMYB2 also localizes to the nucleus, as would be expected for a gene product that controls the transcription of other genes. The full wheat TaMYB73 protein, as well as its C-terminal region, exhibits self-activating ability, but its $\mathrm{N}$-terminal region does not $^{27}$. Previous experiments have shown that the CmMYB19 protein exhibits no transcriptional activation activity when its encoding gene is expressed in yeast ${ }^{28}$. Here, the outcome of a yeast one-hybrid experiment demonstrated that CmMYB2 similarly failed to exhibit transcriptional activation, while its product was suggested to be able to act as a transcriptional repressor when the gene was expressed in A. thaliana protoplasts.

\section{Involvement of CmMYB2 in the control of flowering time}

To transition from the vegetative to the reproductive state, higher plants need to integrate particular environmental signals (such as the photoperiod) with specific endogenous stimuli (such as GA) ${ }^{29,30}$. Both the photoperiod and the GA pathway, which strongly control the
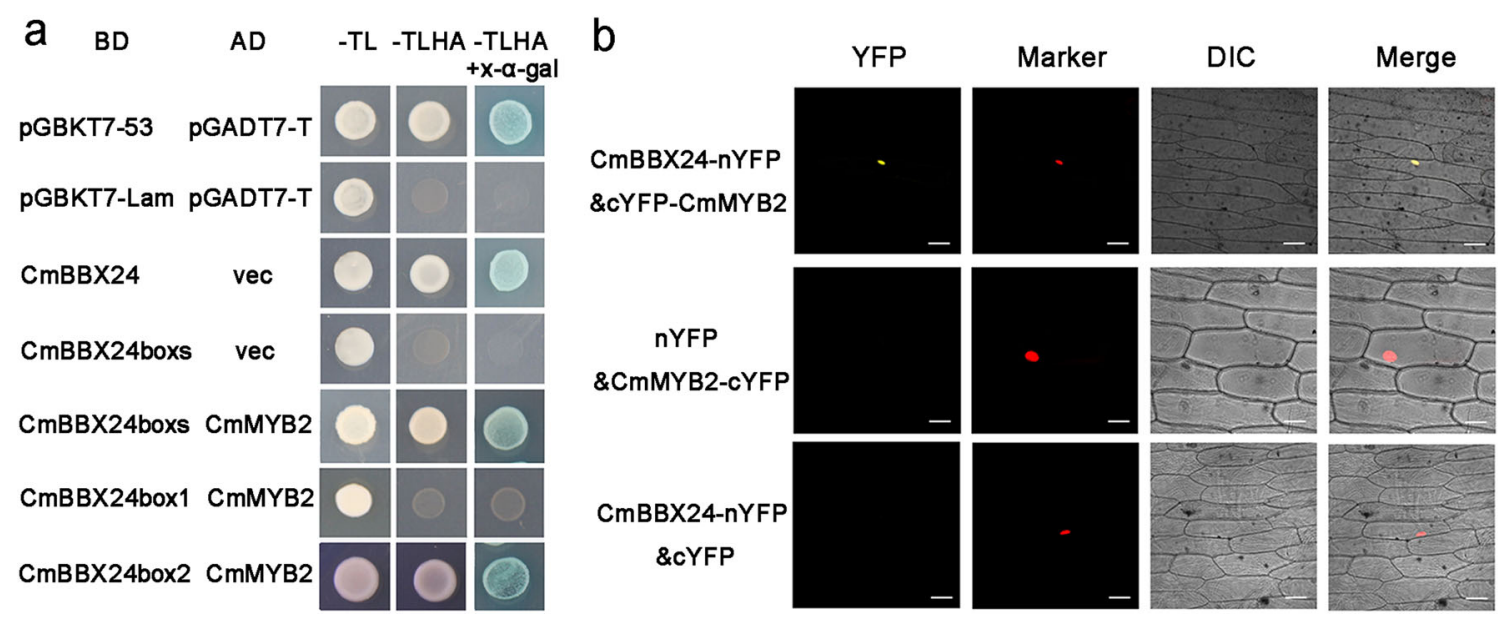

Fig. 5 CmMYB2 interacts with CmBBX24 both in vitro and in vivo. a A yeast two-hybrid assay showing protein interactions. $-\mathrm{TL}$, SD/-Trp/-Leu; -TLHA, SD/-Trp/-Leu/-Ade/-His; -TLHA + X-a-gal, SD/-Trp/-Leu/-Ade/-His/X-a-gal; vec, empty vector; CmBBX24boxs, two B-boxes in the N-terminus of CmBBX24; CmBBX24box1, the first B-box in the N-terminus of CmBBX24; CmBBX24box2, the second B-box in the N-terminus of CmBBX24; pGBKT7-53 and PGADT7-T served as positive controls, and PGBKT7-Lam and PGADT7-T served as negative controls. $\mathbf{b}$ A BiFC assay was used to confirm the interaction between CMMYB2 and CmBBX24, given the results of the yeast two-hybrid assay. YFP: yellow fluorescent protein, Marker: nuclear localization shown by RFP activity, DIC: bright field image, Merge: overlay of YFP, RFP and bright field images 
differentiation of flower buds, are highly conserved among plants and have been intensively studied ${ }^{1,31}$. Here, the RNA-seq approach used to profile the transcriptomes revealed that homologs of a number of known components of the various flowering regulatory pathways were identified as being differentially transcribed between WT plants and those in which the level of expression of $C m M Y B 2$ was either increased or decreased; among these homologs were the GA synthesis/signaling genes CmGA20ox, CmGA2ox, CmGA3ox, CmGRP, CmGID, and $C m D E L L A$ and the photoperiod pathway genes CmCOL1, CmCOL9, CmPRR7, CmPRR9, and CmCDF1. The strong implication is that CmMYB2 influences flowering time in chrysanthemum by regulating GA and photoperiod pathway genes.

MYB transcription factors have a major impact on plant growth and development, but their contribution to the determination of flowering time is not well understood; much of the relevant data is related to A. thaliana and a small number of crop species. The $M Y B$-related proteins MYB-RELATED PROTEIN 1 (MYR1) and MYBRELATED PROTEIN 2 (MYR2) have been shown to be repressors of flowering in plants maintained under lowlight intensity, since the myrl/myr 2 double mutant flowers earlier than WT does ${ }^{32}$. Moreover, the phenotype resulting from overexpression of MYR1 and MYR2 resembles that of a GA-deficient plant. Here, under shortday conditions, chrysanthemum plants engineered to overexpress CmMYB2 flowered earlier than did WT plants, the latter of which in turn flowered before $C m M Y B 2$ knockdown plants did. Altering the level of $C m M Y B 2$ expression affected the leaf content of three forms of $\mathrm{GA}\left(\mathrm{GA}_{1}, \mathrm{GA}_{19}\right.$, and $\left.\mathrm{GA}_{20}\right)$. CmBBX24 knockdown plants also flower before WT plants, and the transcription of the CmBBX24 gene is affected by the photoperiod; it can also be manipulated by the application of $\mathrm{GA}^{23}$. It was possible to show that CmMYB2 and CmBBX24 interacted both in vitro and in vivo. It is possible that CmMYB2 relieves the CmBBX24 inhibition of the GA pathway based on their interaction and reverse effects on flowering. Unexpectedly, the flowering time of A. thaliana plants constitutively expressing $C m M Y B 2$ is delayed when plants are exposed to long-day conditions ${ }^{22}$. This difference in behavior may be related to the fact that, unlike the short-day plant species Jinba chrysanthemum, flowering of $A$. thaliana is induced by long days. Heterologous transformation of a gene leading to contrasting flowering times has also been reported in wheat and Arabidopsis $^{33}$. The details of the mechanism thus need to be elucidated.

In conclusion, overexpression of the chrysanthemum gene $C m M Y B 2$, which encodes a MYB protein, accelerated flowering, while its suppression delayed flowering. CmMYB2 probably exerts its effect on flowering time through the GA pathway, since the leaf GA content was responsive to the intensity of $C m M Y B 2$ expression. This mode of action is consistent with CmMYB2's ability to interact with $\mathrm{CmBBX} 24$, because the latter protein is also known to regulate flowering through the GA pathway ${ }^{23}$.

\section{Materials and methods \\ Plant materials and cultivation conditions}

Cuttings of the chrysanthemum variety Jinba at the fiveor six-leaf stage were obtained from Nanjing Agricultural University's Chrysanthemum Germplasm Resource Preservation Center (Nanjing, China) and were grown in a 1:1:1 (v/v/v) nutrient-enriched soil:vermiculite:perlite mixture.

\section{Isolation of CmMYB2}

A $1 \mu \mathrm{g}$ aliquot of RNA extracted from the leaves of a Jinba plant using RNAiso reagent (TaKaRa, Tokyo, Japan) was converted into ss cDNA using M-MLV reverse transcriptase (TaKaRa). The full-length CmMYB2 sequence of Jinba was inferred from the version of the sequence present in the variety Zhongshanzigui ${ }^{22}$. The full-length $C m M Y B 2$ cDNA sequence was subsequently inserted into a pMD19-T easy vector (TaKaRa) for validation by sequencing. The relevant primers used for this procedure are listed in Table S1.

\section{Subcellular localization of CmMYB2}

The CmMYB2 open reading frame was amplified using the primer pair CmMYB2-pENTR1A-F/-R, which ensured that the amplicon carried a SalI recognition site at its $5^{\prime}$ end and a NotI recognition site at its $3^{\prime}$ end (the primer details are listed in Table S1). The resulting amplicon was inserted into a pENTR1A vector (Invitrogen, Carlsbad, CA, USA) and subsequently into a pMDC43 vector ${ }^{34}$ using a reaction based on LR Clonase II (Invitrogen). The CmMYB2 sequence was fused to the Nterminus of GFP, and the fusion construct was placed under the control of the CaMV $35 \mathrm{~S}$ promoter, forming a p35S::GFP-CmMYB2 construct. This construct (or, as a control, p35S::GFP) was transformed into onion epidermal cells using a PDS-1000 He-driven particle bombardment device (Bio-Rad, Hercules, CA, USA). The bombarded tissue was cultivated in the dark at $23^{\circ} \mathrm{C}$ for $16 \mathrm{~h}$ on Murashige and Skoog (MS) media ${ }^{35}$ before being assayed for GFP activity using confocal laser scanning microscopy (LSM780, Zeiss, Oberkochen, Germany).

\section{Analysis of the transcriptional activity of CmMYB2}

The CmMYB2 open reading frame was amplified using the primer pair MYB2-BD-F/-R (see Table S1 for details), which added an $\mathrm{NdeI}$ recognition site to the $5^{\prime}$ end and a BamHI recognition site to the $3^{\prime}$ end. After purification, 
the amplicon was inserted into a pGBKT7 vector (Clontech, Mountain View, CA, USA). A pCL1 vector containing a full-length copy of GAL4 served as a positive control, and an empty pGBKT7 plasmid served as a negative control. The constructs were transformed into bakers' yeast (Saccharomyces cerevisiae) strain Y2HGold (Clontech) following the "Yeast Transformation System 2" protocol provided by the manufacturer. The pCL1 transformants were incubated on SD/leucine dropout media, while the pGBKT7 and pGBKT7-CmMYB2 transformants were incubated on $\mathrm{SD} /$ tryptophan dropout media. After culturing at $30^{\circ} \mathrm{C}$ for three days, the transformed cell lines were transferred to SD histidine and adenine dropout media.

Luminescence assays were used to detect the transactivation activity of $C m M Y B 2$ in A. thaliana mesophyll protoplasts. The pENTR1A-CmMYB2 construct was previously transformed into $\mathrm{p} 35 \mathrm{~S}:: G A L 4 D B$ vectors using a reaction based on LR Clonase II. Transient expression of the transgene was achieved as described previously ${ }^{36}$. A $7.5 \mu \mathrm{g}$ aliquot of either p35S::GAL4DB-AtARF5 (positive control), p35S::GAL4DB (negative control) or p35S:: GAL4DB-CmMYB2 (test construct) was introduced into the protoplasts. A $7.5 \mu \mathrm{g}$ aliquot of the luciferase (LUC) reporter construct GAL4-LUC was then cotransformed into $A$. thaliana mesophyll protoplasts. LUC assays were performed as described previously ${ }^{37}$, except that Dluciferin was replaced by ViviRen Live Cell substrate (www.goldbio.com/). A. thaliana mesophyll protoplasts were cultivated in plates for $16 \mathrm{~h}$ in the light at $23^{\circ} \mathrm{C}$. A low-light cooled CCD imaging apparatus (DU934P, Andor, UK) was used to capture LUC images from a 96well plate. The LUC activity was measured in $10 \mathrm{~s}$ intervals, and the luminescence counts were quantified using a 20/20n luminometer (Turner Biosystems Inc., Sunnyvale, CA, USA). Three independent experiments were performed for each assay.

\section{Generation and characterization of chrysanthemum transgenics}

The $C m M Y B 2$ open reading frame sequence was amplified using primer pair CmMYB2-pBIG-F/R (details given in Table S1), which has a BamHI recognition site at its $5^{\prime}$ end and a SacI recognition site at its $3^{\prime}$ end, and the resulting amplicon was inserted into a pBIG vector (in which the restriction enzyme sites were reconstructed $)^{38}$. The primer sequences required for the CmMYB2 RNAi fragment were retrieved from wmd3.weigelworld.org. The fragments required to knock down $C m M Y B 2$ by RNAi were obtained by following an artificial microRNA cloning protocol (wmd3.weigelworld.org/cgi-bin/webapp ${ }^{39}$ ) and were inserted adjacent to the plasmid's BamHI and SmaI recognition sites (Table S1); the plasmid was subsequently transferred into the pBIG vector.
The overexpression and RNAi transgenes were inserted into Agrobacterium tumefaciens EHA105 using the freezing transformation method, after which the transformed bacteria were inserted into Jinba plants via Agrobacterium-mediated transformation ${ }^{40}$. The growth and development of the two most effective overexpressing (OX-1 and -2) and knockdown (RNAi-1 and -2) plants were assessed at various time points. Flower bud differentiation was divided into five stages: the FBD stage, visible color (VC) stage, EO stage, open-flower (OF) stage and senescent-flower (SF) stage.

\section{RNA-seq analysis}

OX-1, RNAi-2, and WT plants were grown under controlled conditions $\left(23^{\circ} \mathrm{C}, 60 \%\right.$ relative humidity, and a $16 \mathrm{~h}$ photoperiod provided by $150 \mu \mathrm{mol} \mathrm{m}^{-2} \mathrm{~s}^{-1}$ illumination) for four weeks. Their third fully expanded leaf and stem apical meristem were harvested at 8 a.m. (after three hours of light exposure), flash frozen in liquid nitrogen and then stored at $-80^{\circ} \mathrm{C}$ until needed. RNA was extracted from these explants using RNAiso reagent (TaKaRa). RNA-seq was performed on pooled RNA formed by combining equal quantities; the sequencing was performed by a HiSeq 2000 device (Illumina, San Diego, CA, USA) housed at the Beijing Genomics Institute (Shenzhen, China). Adapter contamination sequences (match length $\geq 10 \mathrm{bp}$ ), low-quality reads and noncalled bases were discarded to obtain a set of clean reads for use in subsequent bioinformatic analyses. The transcriptomes were assembled into contigs using the Trinity program ${ }^{41}$, and the resulting unigenes were annotated using the Kyoto Encyclopedia of Genes and Genomes (KEGG) (www.genome.jp/kegg/kegg1.html), NCBI nonredundant protein (NR) (www.ncbi.nlm.nih. gov/refseq), NCBI nonredundant nucleotide (NT) (www. ncbi.nlm.nih.gov/nuccore), Swiss-Prot (www.uniprot.org), Cluster of Orthologous Groups (COG) (www.ncbi.nlm. nih.gov/COG/) and Gene Ontology (GO) (geneontology. org/page/go-database) databases. Differentially transcribed genes were subjected to both GO functional and KEGG pathway analyses.

\section{Quantitative real-time PCR (qRT-PCR) analysis}

Total RNA was extracted using RNAiso reagent (TaKaRa), treated with DNase to remove contaminating genomic DNA and reverse-transcribed using M-MLV reverse transcriptase. The resulting ss cDNA served as a template for a series of qRT-PCRs in which each reaction mixture consisted of $10 \mu \mathrm{L}$ of SYBR Premix Ex Taq ${ }^{\mathrm{TM}}$ II (TaKaRa), $0.4 \mu \mathrm{L}$ of each primer $(10 \mu \mathrm{M})$ and $5 \mu \mathrm{L}$ of $1 \mathrm{ng} /$ $\mu \mathrm{L}$ template. The chrysanthemum EF1 $\alpha$ gene (KF305681) served as a reference. The $2^{-\Delta \Delta C t}$ method was used to calculate the relative abundance of the various transcripts, and each qRT-PCR was carried out in triplicate. 


\section{Quantifying the GA content in chrysanthemum leaves}

WT, OX. and RNAi plants were grown under an 8-h photoperiod for 30 days. Three of the youngest, fully opened leaves $(0.5 \mathrm{~g}$ of fresh tissue) were harvested after they had been exposed to $3 \mathrm{~h}$ of light, with each line sampled in triplicate. The method used to determine the leaf GA content has been described previously ${ }^{23}$.

\section{Yeast two-hybrid assays}

Each of the sequences of $C m B B X 24, C m B B X 24 b o x s$, $C m B B X 24 b o x 1$, and $C m B B X 24 b o x 2$ were inserted into pGBKT7 vectors (Clontech), while $C m M Y B 2$ was inserted into a pGADT7 vector (Clontech). The primers used are listed in Table S1. The resulting pGBKT7-CmBBX24boxs, pGBKT7-CmBBX24box1, and pGBKT7-CmBBX24box2 constructs were cotransformed together with pGADT7CmMYB2 into Y2HGold cells (Clontech) according to the "Yeast Transformation System 2" protocol. The transformed cells were subsequently incubated on tryptophan and leucine SD media and on tryptophan, leucine, histidine and adenine SD media in the presence or absence of $\mathrm{X}$ - $\alpha$-gal. pGBKT7-53 and pGADT7-T served as positive controls, and pGBKT7-Lam pGADT7-T served as negative controls.

\section{BiFC assays}

The $C m B B X 24$ and $C m M Y B 2$ open reading frames were inserted into the pSAT4A-nYFP and pSAT4A-cYFP vectors, respectively ${ }^{42}$. The primers used are listed in Table S1. The resulting constructs and the p35S::D53-RFP nuclear marker ${ }^{43}$ were transformed into onion epidermal cells using a PDS-1000He-driven particle bombardment device (Bio-Rad), after which the cells were cultivated on MS media in the dark for $16 \mathrm{~h}$ at $23^{\circ} \mathrm{C}$. YFP and RFP activity was observed using confocal laser scanning microscopy (LSM780, Zeiss, Oberkochen, Germany).

\section{Acknowledgements}

This research was supported by the National Natural Science Foundation of China (31572159), the Jiangsu Modern Agricultural Industry Technology System Construction Project (JATS[2018]278), the Fundamental Research Funds for Central Universities (KJQN201815, KYZ201832), the Priority Academic Program Development of Jiangsu Higher Education Institutions and the Jiangsu Planned Projects for Postdoctoral Research Funds.

\section{Author contributions}

F.D.C., J.F.J, and S.M.C. conceived and designed the research; L.Z., Y.X.G., and Z. H.Z. conducted the experiments; Y.N.L., A.P.S., and M.A.J. analyzed the data; and L.Z. wrote the manuscript. All authors read and approved the final manuscript.

\section{Conflict of interest}

The authors declare that they have no conflict of interest.

Supplementary Information accompanies this paper at (https://doi.org/ 10.1038/s41438-020-0317-1).

Received: 23 August 2019 Revised: 1 April 2020 Accepted: 6 April 2020 Published online: 01 July 2020

\section{References}

1. Fornara, F., De, M. A. \& Coupland, G. SnapShot: control of flowering in Arabidopsis. Cell 141, 550-550e2 (2010).

2. Song, Y. H., Ito, S. \& Imaizumi, T. Flowering time regulation: photoperiod- and temperature-sensing in leaves. Trends Plant. Sci. 18, 575-583 (2013).

3. Mouhu, $\mathrm{K}$. et al. Identification of flowering genes in strawberry, a perennial SD plant. BMC Plant Biol. 9, 122 (2009).

4. Hyun, Y. et al. Multi-layered regulation of SPL15 and cooperation with SOC1 integrate endogenous flowering pathways at the Arabidopsis shoot meristem. Dev. Cell 37, 254-266 (2016).

5. Jung, J. H., Ju, Y., Seo, P. J., Lee, J. H. \& Park, C. M. The SOC1-SPL module integrates photoperiod and gibberellic acid signals to control flowering time in Arabidopsis. Plant J. 69, 577-588 (2012).

6. Oda, A. et al. CSFTL3, a chrysanthemum FLOWERING LOCUS T-like gene, is a key regulator of photoperiodic flowering in chrysanthemums. J. Exp. Bot. 63, 1461-1477 (2012).

7. Wang, Z. Y. \& Tobin, E. M. Constitutive expression of the CIRCADIAN CLOCK ASSOCIATED 1 (CCA1) gene disrupts circadian rhythms and suppresses its own expression. Cell 93, 1207 (1998).

8. Kuno, N. et al. The novel MYB protein EARLY-PHYTOCHROME-RESPONSIVE1 is a component of a slave circadian oscillator in Arabidopsis. Plant Cell 15, 2476-2488 (2003).

9. Tominaga, R. et al. Arabidopsis CAPRICE-LIKE MYB 3 (CPL3) controls endoreduplication and flowering development in addition to trichome and root hair formation. Development 135, 1335-45 (2008).

10. Seo, E., Yu, J., Ryu, K. H., Lee, M. M. \& Lee, I. WEREWOLF, a regulator of root hair pattern formation, controls flowering time through the regulation of $F \mathrm{~m}$ RNA stability. Plant Physiol. 156, 1867-77 (2011).

11. Liu, L. et al. Elevated levels of MYB30 in the phloem accelerate flowering in Arabidopsis through the regulation of FLOWERING LOCUS T. PLOS ONE 9, e89799 (2014).

12. Yan, Y. et al. A MYB-domain protein EFM mediates flowering responses to environmental cues in Arabidopsis. Dev. Cell 30, 437-448 (2014).

13. Abe, M. et al. FE, a phloem-specific Myb-related protein, promotes flowering through transcriptional activation of flowering locus $t$ and flowering locus $t$ interacting protein 1. Plant J. 83, 1059-1068 (2015).

14. Jung, C. et al. Overexpression of AtMYB44 enhances stomatal closure to confer abiotic stress tolerance in transgenic Arabidopsis. Plant Physiol. 146, 623-635 (2008).

15. Liu, S. et al. R2R3 MYB transcription factor PtrMYB192 regulates flowering time in arabidopsis by activating flowering locus c. J. Plant Biol. 56, 243-250 (2013).

16. Zhang, L. et al. The wheat MYB-related transcription factor TaMYB72 promotes flowering in rice. J. Integr. Plant. Biol. 58, 701-704 (2016).

17. Gao, R., Gruber, M. Y., Amyot, L. \& Hannoufa, A. SPL13 regulates shoot branching and flowering time in Medicago sativa. Plant. Mol. Biol. 96, 1-15 (2018).

18. Cheng, P. L. et al. A transcriptomic analysis targeting genes involved in the floral transition of winter-flowering chrysanthemum. J. Plant. Growth. Regul. 37, 1-13 (2017).

19. Ren, L. P. et al. Transcriptomic analysis of differentially expressed genes in the floral transition of the summer flowering chrysanthemum. BMC Genomics 17, 673 (2016).

20. Shinoyama, H., Aida, R., Ichikawa, H., Nomura, Y. \& Mochizuki, A. Genetic engineering of chrysanthemum (Chrysanthemum morifolium): current progress and perspectives. Plant Biotechnol. 29, 323-337 (2012).

21. da Silva, Teixeira et al. Chrysanthemum biotechnology: Quo vadis?. Crit. ev. Plant. Sci. 32, 21-52 (2013).

22. Shan, $H$. et al. Heterologous expression of the Chrysanthemum R2R3-MYB transcription factor CMMYB2 enhances drought and salinity tolerance, increases hypersensitivity to ABA and delays flowering in Arabidopsis thaliana. Mol. Biotechnol. 51, 160-173 (2012).

23. Yang, Y. J. et al. A zinc finger protein regulates flowering time and abiotic stress tolerance in Chrysanthemum by modulating gibberellin biosynthesis. Plant Cell 26, 2038 (2014).

24. Huang, P. et al. OsMYB511 encodes a MYB domain transcription activator early regulated by abiotic stress in rice. Genet. Mol. Res. 14, 9506-17 (2015).

25. Li, H. L., Dong, G. \& Peng, S. Q. Molecular characterization of the Jatropha curcas JCR1MYB1 gene encoding a putative R1-MYB transcription factor. Genet. Mol. Biol. 37, 549 (2014) 
26. Huang, C. et al. NbPHAN, a MYB transcriptional factor, regulates leaf development and affects drought tolerance in Nicotiana benthamiana. Physiol. Plant. 149, 297-309 (2013)

27. He, Y. et al. Ectopic expression of a wheat MYB transcription factor gene TaMYB73, improves salinity stress tolerance in Arabidopsis thaliana. J. Exp. Bot. 63, 1511 (2012).

28. Wang, Y. et al. CmMYB19 Over-expression improves aphid tolerance in chrysanthemum by promoting lignin synthesis. Int. J. Mol. Sci. 18, 619 (2017).

29. Yant, L., Mathieu, J. \& Schmid, M. Just say no: floral repressors help Arabidopsis bide the time. Curr. Opin. Plant. Biol. 12, 580-586 (2009).

30. Wahl, V. et al. Regulation of flowering by trehalose-6-phosphate signaling in Arabidopsis thaliana. Science 339, 704 (2013).

31. Boss, P. K., Bastow, R. M., Mylne, J. S. \& Dean, C. Multiple pathways in the decision to flower: enabling, promoting, and resetting. Plant Cell 16(Suppl), S18-S31 (2004).

32. Zhao, C., Hanada, A., Yamaguchi, S., Kamiya, Y. \& Beers, E. P. The Arabidopsis Myb genes MYR1 and MYR2 are redundant negative regulators of flowering time under decreased light intensity. Plant J. 66, 502-515 (2011).

33. Zhang, Y. et al. The cyclophilin CYP20-2 modulates the conformation of BRASSINAZOLE-RESISTANT1, which binds the promoter of FLOWERING LOCUS D to regulate flowering in Arabidopsis. Plant Cell 25, 2504-21 (2013)
34. Curtis, M. D. \& Grossniklaus, U. A gateway cloning vector set for highthroughput functional analysis of genes in planta. Plant Physiol. 133, 462-469 (2003).

35. Murashige, T. \& Skoog, F. A revised medium for rapid growth and bio assays with tobacco tissue cultures. Physiol. Plant. 15, 473-497 (1962).

36. Wu, F. H. et al. Tape-Arabidopsis Sandwich - a simpler Arabidopsis protoplast isolation method. Plant Methods 5, 16 (2009).

37. Fujikawa, Y. \& Kato, N. Split luciferase complementation assay to study proteinprotein interactions in Arabidopsis protoplasts. Plant J. 52, 185 (2007).

38. Becker, D. Binary vectors which allow the exchange of plant selectable markers and reporter genes. Nucleic Acids Res. 18, 203 (1990).

39. Alvarez, J. P. et al. Endogenous and synthetic microRNAs stimulate simultaneous, efficient, and localized regulation of multiple targets in diverse species. Plant Cell 18, 1134-51 (2006).

40. Cui, X. L., Chen, F. D. \& Chen, S. M. Establishment of regeneration and transformation system of ground-cover chrysanthemum Yuhuaxunzhang. JNAU China 32, 40-46 (2009).

41. Grabherr, M. G. et al. Full-length transcriptome assembly from RNA-Seq data without a reference genome. Nat. Biotechnol. 29, 644-652 (2011).

42. Citovsky, $\vee$. et al. Subcellular localization of interacting proteins by bimolecular fluorescence complementation in planta. J. Mol. Biol. 362, 1120-1131 (2006)

43. Zhou, F. et al. D14-SCF ${ }^{\mathrm{D} 3}$-dependent degradation of D53 regulates strigolactone signalling. Nature 504, 406 (2013). 